\title{
SUB-LUMINOUS BLUE STARS AND WHITE DWARFS
}

Jesse L. GREENSTEIN

Mount Wilson and Palomar Observatories

Sub-luminous stars of high temperature show a wide variety of spectra and physical properties. Vorontsov-Velyaminov has called this region of the HR diagram the "blue-white sequence". I wish to discuss the fainter members of this class. The growth of knowledge of colors and spectra has been rapid compared to that of the luminosities, and unfortunately only rough location in the HR diagram is yet possible. Figure I shows the general location and subdivision of sub-luminous stars. I shall discuss :

(I) Old novae.

(2) Horizontal-branch stars.

(3) Hot subdwarfs.

(4) White dwarfs.

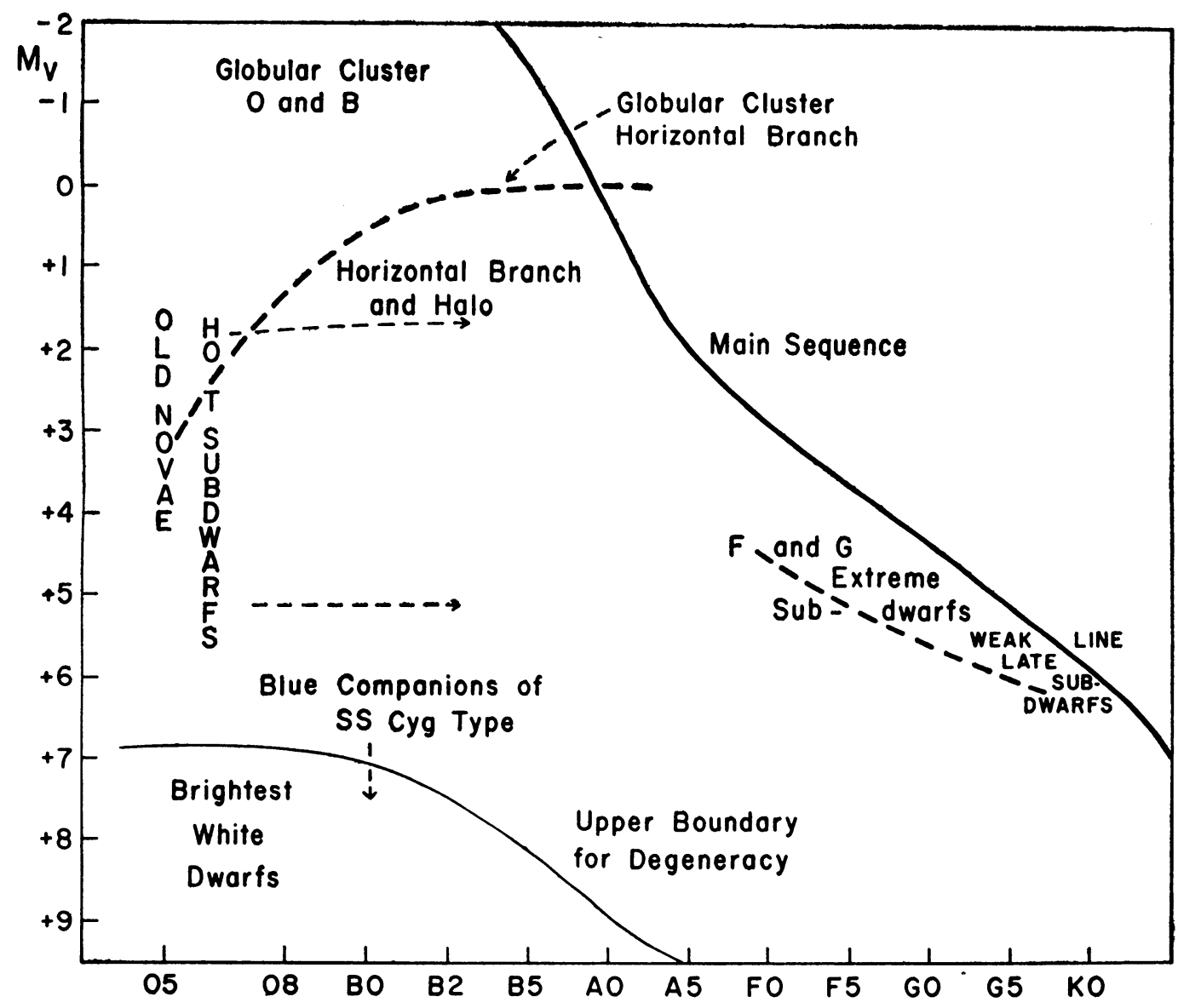

FIGURE I. Location of various types of stars in a schematic HR diagram. 
I. Old Novae. The range of visual luminosity is approximately from +2 to +6 . Their spectra, as observed at Palomar, generally show weak, relatively narrow emission lines and no absorption lines at an epoch up to roo years after the outburst. The post-nova ejection of gases may last much longer, but from the absence of such emission lines among the many other faint blue stars so far investigated, it seems possible that it does not persist for the full interval between nova outbursts. In spite of a wide variety of colors (in part caused by interstellar reddening) the old novae show a strong ultraviolet continuum, maintain high-excitation emission lines and must be intrinsically very hot. Intrinsic reddening (possibly by a Kosirev mechanism in an envelope) is not excluded. No spectral features belonging to a late-type star have been so far observed in single old novae. However novae in binary systems show more complex spectra arising from interactions between the stars.

II. Horizontal-branch Stars. The first spectra obtained by Münch were in globular clusters. Greenstein has found many similar objects among the blue stars of the galactic halo. A preliminary statistical investigation indicates luminosities in the range $\mathrm{o}^{\mathrm{m}}$ to $+2^{\mathrm{m}}$. The photoelectric colors include some of the bluest stars yet known. Spectra show that a peculiar, not yet understood phenomenon produces strong, sharp hydrogen lines and large Balmer jump, and very weak lines of all other elements including $\mathrm{Mg}$ II and $\mathrm{He}$ I. Such objects have been recognized spectroscopically to $\mathrm{I}^{\mathrm{m}} \mathrm{m}$, i.e. to a height of $6000 \mathrm{psc}$ above the galactic plane.

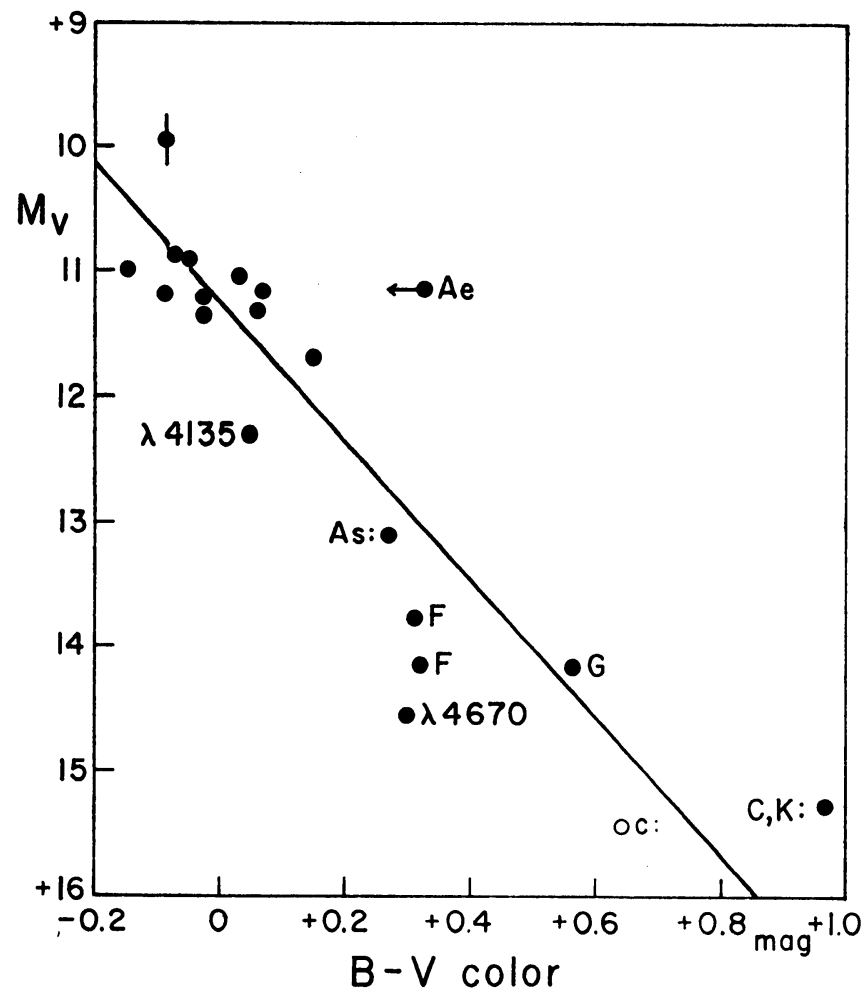

FIGURE 2. HR diagram for white dwarfs of known parallax, photoelectric color and spectrum. Unlabelled solid dots are type DA.
Fainter blue stars are known, and multi-color photoelectric photometry could establish their assignment to this group.

III. Hot Subdwarfs. The first members of this group were recognized by Münch and Greenstein; at present 7 stars of type $\mathrm{O}$ and 5 of type $\mathrm{B}$ can be placed in this group spectroscopically. The brightest are near $+2^{\mathrm{m}}$, the faintest about $+5^{\mathrm{m}}$; nothing is known about the dependence of luminosity on type. Parallaxes are badly needed, especially since some of these may merge with the white dwarfs. Spectra reveal very high temperature and pressure and low rotation. Hydrogen is weak and shallow and the Balmer jump is small. Helium and nitrogen show high relative abundances.

IV. White Dwarfs (see Pl. I, p. 38). The white dwarfs are now being studied extensively. The HR 
diagrams are shown in Figures 2 and 3, with only data of highest reliability shown in Figure 2. The luminosity depends closely on the color, even within the large group classed as DA (hydrogen lines). Earlier classifications showed only that the hydrogen lines persisted over a wide range

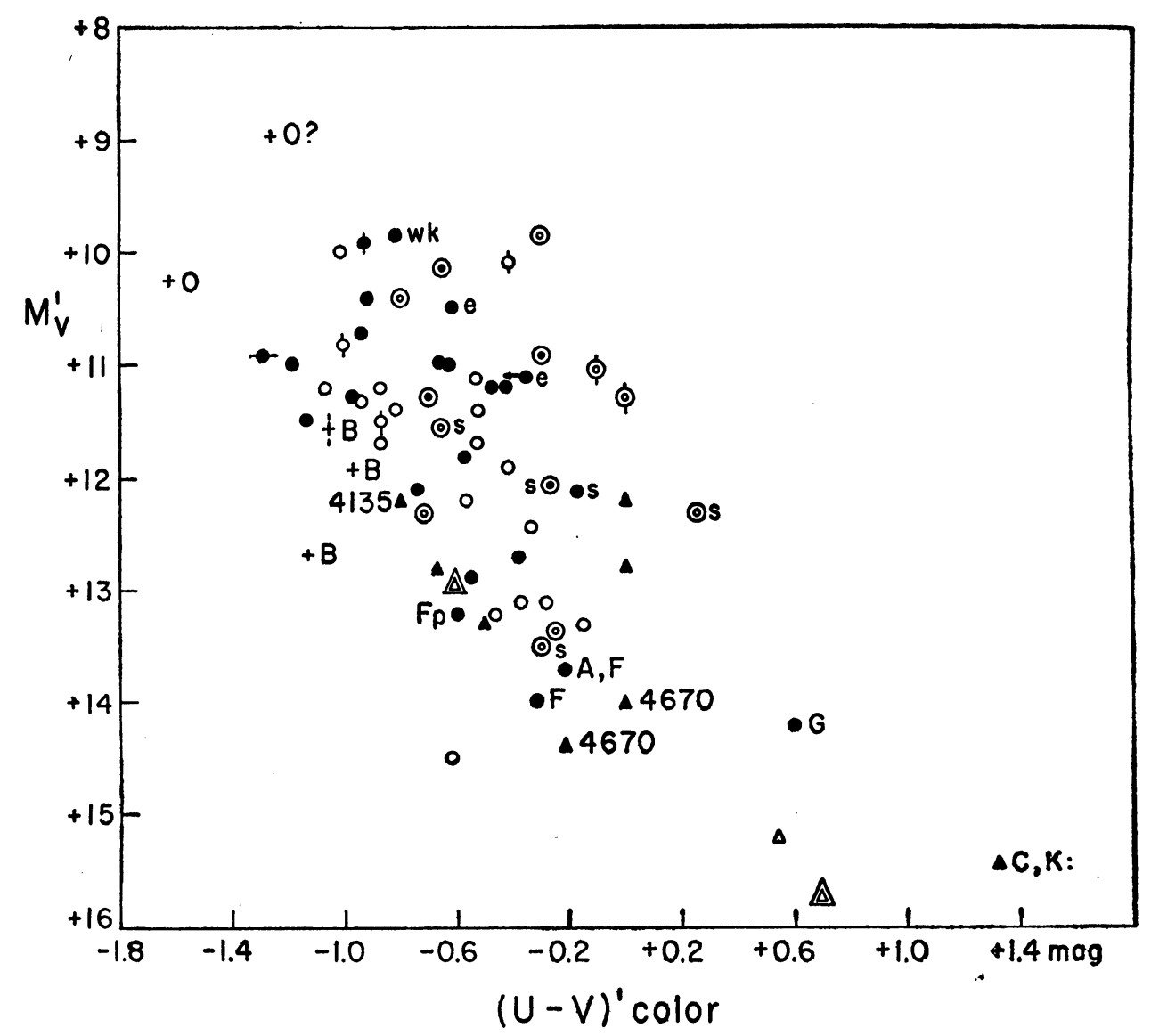

FIGURE 3. HR diagram for white dwarfs. Many luminosities from proper motions only and color deduced from photographic work. Symbols : - solid dots DA, DF, DG spectra by Greenstein, photoelectric colors; solid triangles $\mathrm{DC}, \lambda_{4} \mathrm{I}_{35}, \lambda_{4} 670$ types; crosses types DB or DO. Open symbols spectra by others; ringed solid or open symbols have only Luyten color, or poor luminosities or both.

of color. The present spectrophotometric results show that the hydrogen varies with color, and has a sharp maximum. Sub-classification of type DA is possible using the equivalent widths.

As the white dwarfs become redder, more advanced spectral types are found correlated with decreasing luminosity. The color-spectrum relation is not unique; stars with essentially continuous spectra are found throughout the sequence. Variations in surface composition almost certainly exist. However, analysis of photoelectric colors and spectra provide a reasonable temperature scale, ranging from $40,000^{\circ}$ to $4,000^{\circ}$. The mean radius is $0.013 \mathrm{R} \odot$, mean mass $0.56 \mathrm{M} \odot$ (for zero hydrogen content), and the real scatter is very small. The total deviation of radius, including errors of observation and interpretation, is by a factor of two. 
The variety of faint blue objects now found makes it implausible that all stars follow a unique course of evolution towards the white dwarf stage. With normal masses and internal density distribution most faint blue stars need not be degenerate and may still possess thermonuclear energy sources. The subluminous stars are largely very hot, suggesting that contraction occurs after energy sources near exhaustion. The majority of objects here described belong to population II (the spherical sub-system) although observational selection by discovery in the galactic polar cap plays some part. White dwarfs are clearly members of all stellar populations. Halo, subdwarf and white dwarf stars show changes in the abundances of certain elements, caused by thermonuclear processes. Extended envelope phenomena may also occur in some of these stars.

The white dwarfs start at an absolute magnitude well below that of most subdwarfs or old novae, and form a relatively simple sequence. Without energy sources or the possibility of contraction they will cool off slowly and travel downward to the right along a track set by the initial mass. The age of the redder white dwarfs near $\mathrm{M}=+\mathrm{I}_{5}$ must be about 8 billion years. The low average mass, $0.6 \mathrm{M} \odot$, requires that mass loss be very substantial. In fact old stars that are now white dwarfs must have had masses greater than $\mathrm{I} .4 \mathrm{M} \odot$ and have lost about 60 percent of their mass.

\section{Discussion}

Eminzadé, Remarks on the Chemical Composition and Energy Sources of White Dwarfs. From Chandrasekhar's theory it follows that the hydrogen abundance in white dwarfs constitutes tens of percent by weight, i.e. that it is of the same order as in other stars. But the thermonuclear reactions would be very intense in white dwarfs, if the hydrogen abundance were so high. In order to produce the observed low luminosity the abundance of hydrogen must be inappreciably small, only some millionths parts of a percent. The opinion about the absence of hydrogen in white dwarfs is generally accepted. But we believe this point of view to be incorrect, for the following reasons :

I) The theoretical radii of white dwarfs calculated on the assumption that there is no hydrogen in them gives always considerably smaller results as compared with the observed radii.

2) Hydrogen lines are present in the spectra of white dwarfs. It is unclear why hydrogen exists only in the surface layers of these stars, being absent in their interiors.

3) There are a number of questions on the evolution and energy sources of white dwarfs, which are also unclear.

We believe therefore that white dwarfs are not deprived of hydrogen and that hydrogen must be present in their composition. For the solution of this question, the low luminosity of white dwarfs, in spite of the supposed considerable abundance of hydrogen, must be explained. The rate of thermonuclear reactions grows with increasing density. This is true at a low density, if no degeneracy occurs. The rate of thermonuclear reactions falls at a very high density. This point of view must be accepted proceeding from the fact that white dwarfs have a high density and a very low luminosity. This is an observational result.

At a very high density the properties of matter in the interiors of stars are similar to those of solid bodies. As a result of interaction forces there exists a certain "bond" between the protons, as in a solid (or liquid) body. This prevents the protons from approaching each other closely, and no reactions can therefore be produced.

Thus we can find a way to overcome the serious contradictions existing in the problem of energy sources and the chemical composition of white dwarfs. Other questions about these extremely interesting stars have therefore also been answered. 
Schatzman : Wants to draw the attention of Eminzadé to his book on White Dwarfs, where, he thinks, he can find the answer to most of his questions.

Greenstein : I do not believe that there are any definite discrepancies between observed and theoretical radii. The exhaustion of hydrogen in at least some white dwarfs is a definite observed fact, the helium-rich and continuum types of white dwarfs. No energy source is required to maintain the observed luminous output of the white dwarfs; conduction from the interior is sufficient in some cases up to $\mathrm{IO}^{10}$ years.

Van den Bergh : In connection with Prof. Greenstein's talk it is of interest to note that the clusters NGC 188 with age $\approx 7 \times 10^{\circ}$ years may contain a subluminous $\mathrm{OB}$ star. If the star is a cluster member $\mathrm{M}_{\mathrm{v}} \approx+5, \mathrm{~B}-\mathrm{V} \approx-0.3$.

Greenstein : I am very interested to know that a possible B subdwarf exists in NGC I88. I have suspected that some hot subdwarfs belong to the old population I, and I would be interested in observing it, since it would provide a luminosity for the first time. 
Caption for the plate

FIGURE 4. Palomar spectra of white dwarfs. 


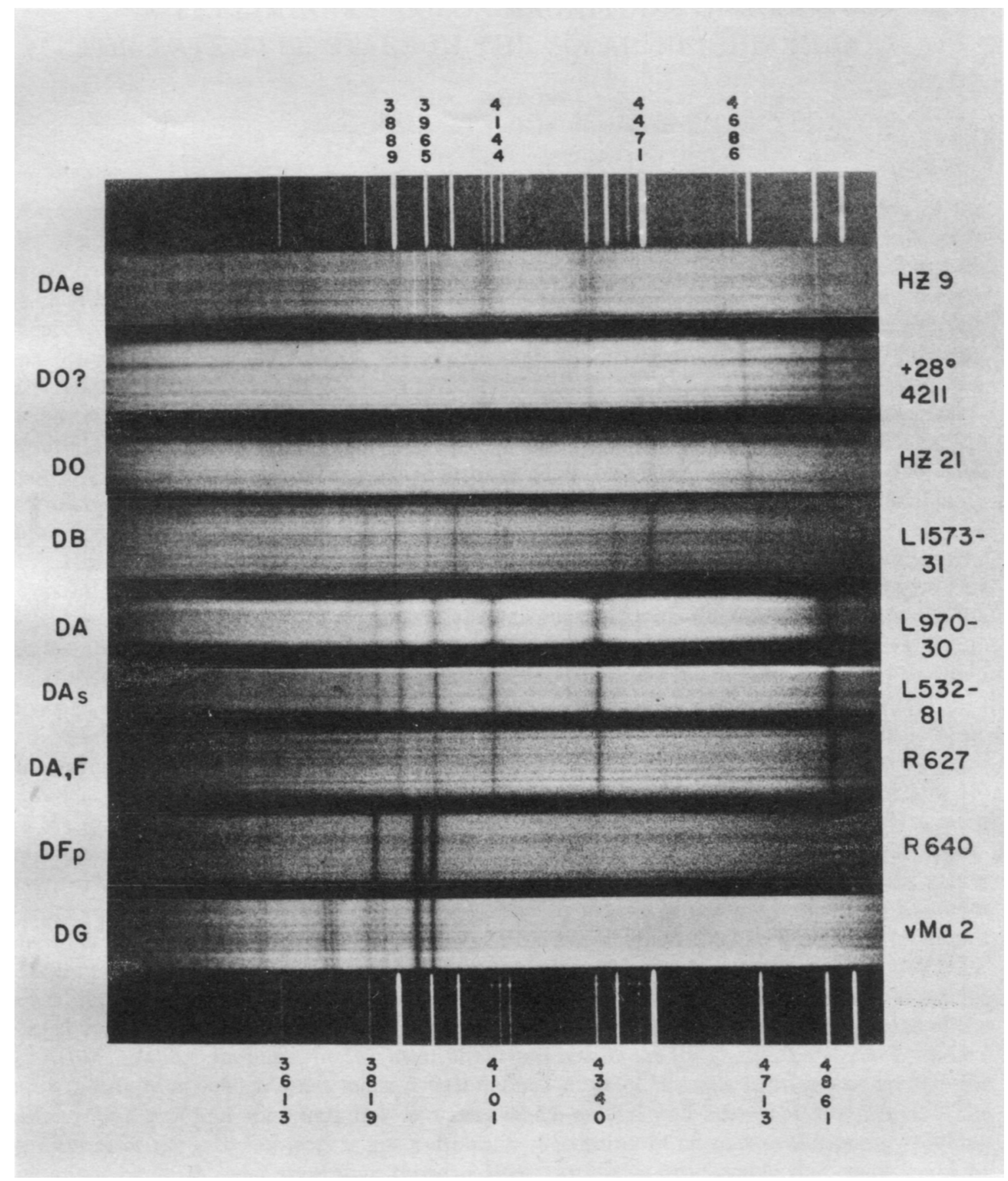

Fig. 4 